\title{
ATP5B and ETFB metabolic markers in children with congenital hydronephrosis
}

\author{
QI ZHAO, YI YANG, CHANGLIN WANG, YING HOU and HUI CHEN
}

\begin{abstract}
Department of Pediatric Surgery, Shengjing Hospital of China Medical University, Shenyang, Liaoning 110004, P.R. China
\end{abstract}
Received September 21, 2015; Accepted October 3, 2016

DOI: $10.3892 / \mathrm{mmr} .2016 .5914$

\begin{abstract}
Congenital obstructive nephropathy is the primary cause of chronic renal failure in children. Disorders of mitochondrial energy metabolism may be a primary factor underlying tubular cell apoptosis in hydronephrosis. The $\beta$-F1-ATPase (ATP5B) and electron transfer flavoprotein $\beta$ subunit (ETFB) metabolic markers are involved in mitochondrial energy metabolism in other diseases. The aim of the present study was to evaluate whether ATP5B and ETFB are represented in the hydronephrotic kidney, and whether they are associated with the progression of hydronephrosis. The cohort examined consisted of 20 children with hydronephrosis, graded III and IV using the Society for Fetal Urology grading system, and a control group consisting of 20 patients with nephroblastoma. Reverse transcription-quantitative polymerase chain reaction and immunoblot analyses were used to investigate the differential expression of genes and proteins in the two groups. The gene and protein expression levels of ATP5B and ETFB were upregulated in the hydronephrosis group. Correlation analyses revealed negative correlations between ATP5B, ETFB protein and split renal function (SRF). Receiver-operator curve analysis found a diagnostic profile of the ETFB protein in identifying children with hydronephrosis with abnormal SRF $(<45 \%)$. These results suggested that increasing levels of ATP5B and ETFB were associated with worsening renal injury. ATP5B and ETFB may be novel markers in hydronephrosis and require further detailed investigation.
\end{abstract}

\section{Introduction}

Unilateral ureteral obstruction (UUO), which results in hydronephrosis, is characterized by decreased renal function, increased interstitial fibrosis, tubular apoptosis and cellular

Correspondence to: Dr Yi Yang, Department of Pediatric Surgery, Shengjing Hospital of China Medical University, 36 Sanhao Street, Heping, Shenyang, Liaoning 110004, P.R. China

E-mail: yangy2@sj-hospital.org

Key words: $\beta$-F1-ATPase, electron transfer flavoprotein $\beta$ subunit, renal tissue, mitochondria, hydronephrosis infiltration $(1,2)$. Histological studies have suggested that the mechanical stretch of the tubular epithelium and oxidative stress are early stress factors, leading to tubular cell injury and death $(2,3)$. There is increasing evidence that the mitochondrial pathway is involved in stretch-induced and oxidative stress-enhanced tubular cell apoptosis $(4,5)$. Therefore, it appears that disorders of mitochondrial energy metabolism may also be a primary factor underlying tubular cell apoptosis in hydronephrosis.

As the kidney is a high energy-demanding organ, it relies heavily on aerobic metabolism for the production of ATP by oxidative phosphorylation within mitochondria. The reprogramming of energy metabolism has been designated a 'new' hallmark for diverse forms of chronic kidney disease (4). Mitochondrial $\beta$-F1-ATPase (ATP5B), the catalytic subunit of the rate-limiting enzyme of oxidative phosphorylation, and electron transfer flavoprotein $\beta$ subunit (ETFB), an electron acceptor of five isoforms of acyl-CoA dehydrogenase, are metabolic markers, which have been shown to be of value in several types of cancer (6) and multiple acylCoA dehydrogenation deficiency (MADD) (7). However, the expression and alterations in these two proteins, and their underlying involvement of in hydronephrosis have not been reported and remain to be elucidated.

In the present study, the mRNA and protein expression levels of ATP5B and ETFB were investigated in cases of hydronephrosis graded III and IV using the Society for Fetal Urology (SFU) grading system, to identify the associations with split renal function (SRF), and thereby evaluate whether ATP5B and ETFB have functions in renal injury and the progression of hydronephrosis.

\section{Materials and methods}

Patients. Following approval by the Ethics Committee of China Medical University (Shenyang, China; no. 2012 PS81K), fresh kidney specimens were prospectively collected from patients undergoing surgery for unilateral hydronephrosis secondary to UUO. The patients underwent surgery at Shengjing Hospital of China Medical University between May 2012 and October 2013. No patients had a urinary tract infection prior to surgery or any other associated anomalies, including vesicoureteral reflux and ureterovesical junction obstruction. A total of 20 control renal biopsy specimens were obtained from patients undergoing nephrectomy for nephroblastoma, and the tissues were confirmed histologically to be unaffected. 
SRF of patients. A technetium-99 m diethylenetriamine pentaacetic acid renal scan (InfiniaVCII Hawkeye; GE Healthcare Life Sciences, Chalfont UK) was used to evaluate the mean renal function. The furosemide clearance half-time in diuretic renography was applied to determine whether obstruction was present. A drainage clearance half-time of $>20$ min was indicative of obstruction. The differential glomerular filtration rate (GFR) of the affected dilated kidney was determined to measure the SRF of the patient. Clearance half-time and GFR were automatically determined by Xeleris image processing system (GE Healthcare Life Sciences). It detected the time-intensity curve and GFR was calculated accordingly. Any cases of 'supranormal function' of the affected kidney (SRF $>55 \%$ ) were omitted from the experimental group.

Tissue sample collection and RNA extraction. Hydronephrotic tissues and normal tissues $\left(10 \mathrm{~mm}^{3}\right)$ were collected, respectively, and identified by pathologists. Various histological changes within the obstructed tissue were present, including visible dilated tubules predominantly located in collecting tubules and other distal tubules, flattened tubular epithelial cells, expansive Bowman capsules and glomerular infiltration by inflammatory cells. In the control group, no abnormalities in tumor cell infiltration were found. Following confirmation, each tissue specimen was examined via molecular analysis, including immunoblot analysis and reverse transcription-quantitative polymerase chain reaction (RT-qPCR) analysis. TRIzol reagent (Invitrogen; Thermo Fisher Scientific, Inc., Waltham, MA, USA) was used for RNA extraction. Following harvesting, the tissue was cut into small pieces on ice and $500 \mu 1$ TRIzol was added. Subsequently, the mixture was homogenized intermittently on ice and processed according to the instructions of the manufacturer. The RNA quality was assessed by measuring A260/A280 using a spectrophotometer. An adequate quantity ( $\geq 3 \mu \mathrm{g}$ ) and quality (A260/A280: 1.8-2.1) of RNA was extracted and used for cDNA synthesis with the Takara RNA PCR kit (Takara Bio, Inc., Otus, Japan).

qPCR analysis. The qPCR amplifications were performed in a $10 \mu 1$ reaction system with $5 \mu \mathrm{l}$ SYBR Premix Ex Taqll (Tli RNaseH Plus; Takara Bio, Inc.), $2 \mu 1 \mathrm{ddH}_{2} 0,0.5 \mu \mathrm{l}$ each primer and $2 \mu \mathrm{l}$ cDNA. Reactions were performed in triplicate on a Light Cycler (Roche Applied Science, Penzberg, Germany) with the primers as follows: Human ATP5B, sense 5'-TCA CCC AGG CTG GTT CAGA-3' and antisense 5'-AGT GGC CAG GGT AGG CTG AT-3'; Human ETFB, sense 5'-AGG AGG TAC TGT CAA AGC TGC-3' and antisense, 5'-TTT CAT GGC CTT CCT CAG GG 3'; Human $\beta$-actin, sense 5' AGA GCT ACG AGC TGC CTG AC-3'and antisense 5'-AGC ACT GTG TTG GCG TAC AG-3'. The housekeeping gene. $\beta$-actin (cat. no. DR3783; Takara Bio, Inc.) was used as an endogenous control. The amplification protocol involved $5 \mathrm{sec}$ denaturation at $95^{\circ} \mathrm{C}$ and $20 \mathrm{sec}$ annealing at $56^{\circ} \mathrm{C}$ for 45 cycles. The relative mRNA levels for each sample were calculated using the $2^{-\Delta \Delta \mathrm{Cq}}$ method (8).

Immunoblot analysis. The tissue specimens $(\sim 50 \mathrm{mg})$ were minced into small sections using surgical blades and sonicated in protein lysis buffer. Proteins were quantified using the 2D Quant kit (GE Healthcare Life Sciences) according to the manufacturer's instructions. Briefly, $50 \mu \mathrm{g}$ of total protein was electrophoretically separated on a $12 \%$ SDS-PAGE gel. The proteins were then transferred onto a PVDF membrane and blocked with $5 \%$ non-fat milk in TBS with $0.1 \%$ Tween 20 (TBST) and shaken for $1 \mathrm{~h}$. The membrane was washed three times with TBST, each wash used $10 \mathrm{ml}$ TBST and lasted $\sim 10 \mathrm{~min}$. The membrane was probed with the following polyclonal antibodies: Anti-ATP5B (1:2,000; monoclonal mouse anti-human; cat. no. ab14730; Abcam, Cambridge, UK) and anti-ETFB (1:500; polyclonal rabbit anti-human; cat. no. ab97036; Abcam ). $\beta$-actin (1:500; monoclonal mouse anti-human; cat. no. sc-8432; Santa Cruz Biotechnology, Inc., Dallas, TX, USA) was used as a loading control. The membranes were incubated with primary antibodies overnight at $4^{\circ} \mathrm{C}$. The protein expression was visualized by incubation with goat anti-mouse (cat. no. sc-3793) and goat anti-rabbit (cat. no. sc-3837) secondary antibody conjugated with horseradish peroxidase (1:2,000; Santa Cruz Biotechnology, Inc.) for $2 \mathrm{~h}$ at room temperature and then enhanced chemoluminescence reagent (Pierce; Thermo Fisher Scientific, Inc.). The intensity of protein staining was determined using Image $2 \mathrm{x}$, a publicly available Java-based image processing program (version 2.1.4.7; National Institutes of Health, Bethesda, MA, USA). The relative density of each protein was calculated by dividing the optical density value of each protein by that of the loading control.

Statistical analysis. All statistical analyses were performed using SPSS 18.0 (SPSS, Inc., Chicago, IL, USA) software for Windows. All values are presented as the mean \pm standard error of the mean. Two-tailed Student's $t$-test was used for analyzing significant differences from the control group. The receiver operating characteristic (ROC) curve was used to determine the cut-off values of ATP5B and ETFB at the optimum sensitivity and specificity. Correlation analysis was used for the comparisons between ATP5B, ETFB and other variables. Bivariate analysis was presented as Pearson or Spearman correlation coefficient. $\mathrm{P}<0.05$ was considered to indicate a statistically significant difference.

\section{Results}

Clinical parameters of patients with hydronephrosis. The clinical and pathological characteristics of the patients are presented in Table I. In the present study, boys were more frequently affected with hydronephrosis, compared with girls, which has also been reported previously (9). The left kidney was more commonly involved than the right kidney (18:2, respectively). The mean age at pyeloplasty was $\sim 2$ years. Grade III hydronephrosis was present in six children, and grade IV hydronephrosis was present in 14 children. The AP pelvic diameters and SRF in the study group are listed. A radionuclide scan was not performed in children from the control group; therefore, the SRF was not evaluated in this group.

Transcription levels of ATP5B and ETFB. Compared with the normal kidney tissues, the kidney tissues with hydronephrosis showed increased mRNA levels of ATP5B, by 2.57-fold $(\mathrm{P}<0.05$; Table II). The mRNA expression of ETFB did not 
Table I. Summary of the clinical parameters of all patients included in the present study.

\begin{tabular}{lcc}
\hline Clinical parameter & Hydronephrosis & Normal control \\
\hline Gender, n (male/female) & $20(14 / 6)$ & $20(12 / 8)$ \\
Age at the time of surgery (years) & $2.16 \pm 2.88$ & $2.56 \pm 1.09$ \\
Clinical diagnosis (SFU grading) & 6 \\
Grade III & 14 \\
Grade IV & $18 / 2$ & - \\
Laterality (left/right) & $35.00 \pm 17.72$ & - \\
Split renal function (\%) & $37.35 \pm 11.40$ & - \\
AP at prepyeloplasty (mm) & & - \\
\hline
\end{tabular}

Data are presented as the mean \pm standard error of the mean. SFU, the Society for Fetal Urology; AP, anteroposterior.

Table II. Relative mRNA level of ATP5B in the two tissue groups.

\begin{tabular}{lccccc}
\hline Tissue section & $\begin{array}{c}\text { ATP5B average } \\
\text { Cq value }\end{array}$ & $\begin{array}{c}\beta \text {-actin } \\
\text { average Cq value }\end{array}$ & $\Delta \mathrm{Cq}$ & $\Delta \Delta \mathrm{Cq}$ & $\begin{array}{c}\text { Fold-change of gene } \\
\text { (vs. normal) }\end{array}$ \\
\hline Hydronephrosis & $24.050 \pm 1.582$ & $24.412 \pm 3.095$ & -0.362 & -1.359 & 2.57 \\
Normal & $24.600 \pm 1.470$ & $23.603 \pm 0.878$ & 0.997 & 0 & 1 \\
\hline
\end{tabular}

ATP5B, $\beta$-F1-ATPase.

Table III. Relative mRNA level of ETFB in the two tissue groups.

\begin{tabular}{|c|c|c|c|c|c|}
\hline Tissue section & $\begin{array}{l}\text { ETFB average } \\
\text { Cq value }\end{array}$ & $\begin{array}{c}\beta \text {-actin average } \\
\text { Cq value }\end{array}$ & $\Delta \mathrm{Cq}$ & $\Delta \Delta \mathrm{Cq}$ & $\begin{array}{c}\text { Fold-change of gene } \\
\text { (vs. normal) }\end{array}$ \\
\hline Hydronephrosis & $23.923 \pm 1.220$ & $24.412 \pm 3.095$ & -0.489 & -0.346 & 1.27 \\
\hline Normal & $23.460 \pm 0.813$ & $23.603 \pm 0.878$ & -0.143 & 0 & 1 \\
\hline
\end{tabular}

ETFB, electron transfer flavoprotein $\beta$ subunit.

A

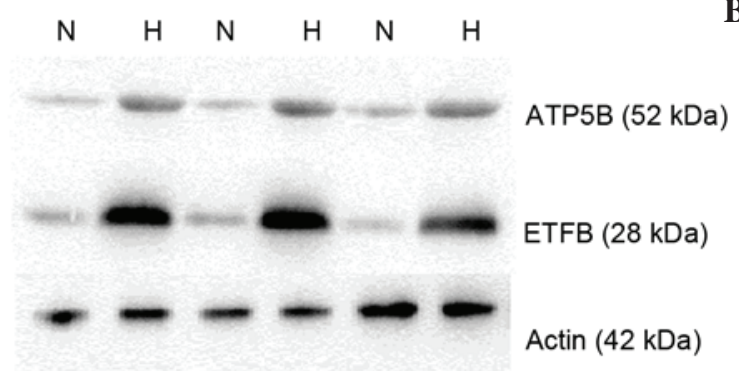

B

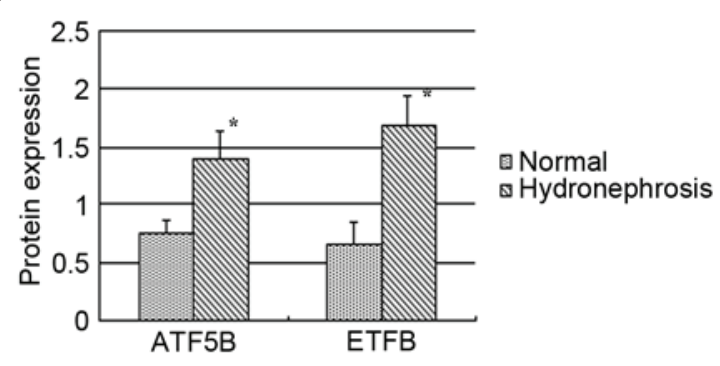

Figure 1. Protein expression of ATP5B and ETFB in hydronephrotic and normal control kidney tissues. (A) Immunoblotting and (B) quantification were performed. $\beta$-actin was used as an internal control. ${ }^{*} \mathrm{P}<0.05$. $\mathrm{H}$, hydronephrosis; $\mathrm{N}$, normal. ATP5B, $\beta$-F1-ATPase; ETFB, electron transfer flavoprotein $\beta$ subunit.

differ between the normal kidneys and those in the hydronephrosis group did not differ in $(\mathrm{P}>0.05)$ (Table III).

Results of immunoblot analysis. Higher protein expression levels of ATP5B and ETFB were observed in the hydronephrosis group (Fig. 1), which were upregulated in the hydronephrotic kidney by 1.87 - and 2.58 -fold, respectively $(\mathrm{P}<0.05)$.

Correlations between ATP5B, ETFB and SRF. A negative correlation was found between the ATP5B protein and SRF $(r=-0.458 ; P=0.042)$. The correlation was even more marked 

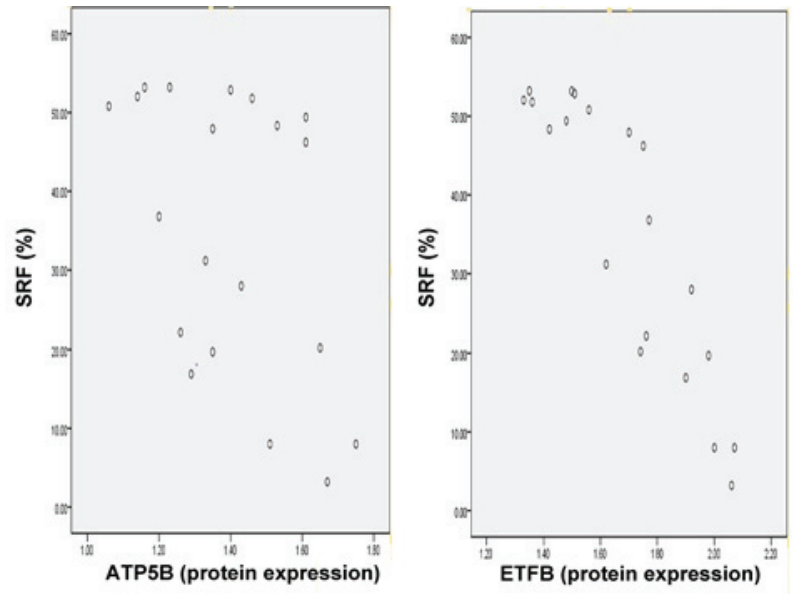

Figure 2. Results of correlation analyses between ATP5B and ETFB protein expression and SRF (\%). ATP5B: $r=-0.458 ; \mathrm{P}=0.042$; ETFB: $r=-0.753$; $\mathrm{P}<0.001$. SRF, split renal function; ATP5B, $\beta$-F1-ATPase; ETFB, electron transfer flavoprotein $\beta$ subunit.

between ETFB protein and SRF ( $\mathrm{r}=-0.753$; P<0.001) (Fig. 2). No statistically significant correlations were found between the mRNA expression of ATP5B and ETFB and SRF. In addition, no significant correlation was found and the age of the patients, parenchymal thickness, GFR and initial AP diameter (length) of the pelvis.

ROC analyses were performed to define the diagnostic profile of ATP5B and ETFB in identifying children with abnormal SRF $(<45 \%)$ among all examined children. In the analysis, it was found that only ETFB showed a suitable diagnostic profile, with the area under the curve (AUC) for the ETFB protein being 0.900 (95\% confidence interval, $0.766-1.034$ ) with an optimal cutoff value of 1.59 (sensitivity, 90\%; specificity, 70\%; Fig. 3).

\section{Discussion}

Obstructive nephropathy is a major cause of renal failure, particularly in infants and children. Despite numerous clinical and experimental studies over several decades, the cellular and molecular mechanisms responsible for the progression of hydronephrosis remain to be fully elucidated (10).

In post-UUO, mechanical stretch of the tubular epithelium and oxidative stress are considered to be of importance and act as early stress factors leading to tubular cell injury. Studies $(11,12)$ have demonstrated that stretch induces caspase-dependent apoptosis in tubular epithelial cells via the intrinsic mitochondrial pathway, and the mitochondrial release of proapoptotic factors into the cytosol leads to apoptosome assembly, the activation of caspase- 9 and the cleavage of effector caspases.

Oxidative stress, which develops from an imbalance between increased free radical production and reduced antioxidant defenses, is considered to be an important pathogenic mechanism in obstructive nephropathy. Perturbations in cellular oxidant handling affect downstream cellular signaling and, in the kidney, promote renal cell apoptosis and senescence, decreased regenerative ability of cells and fibrosis. These characteristics may be caused, at least in part,

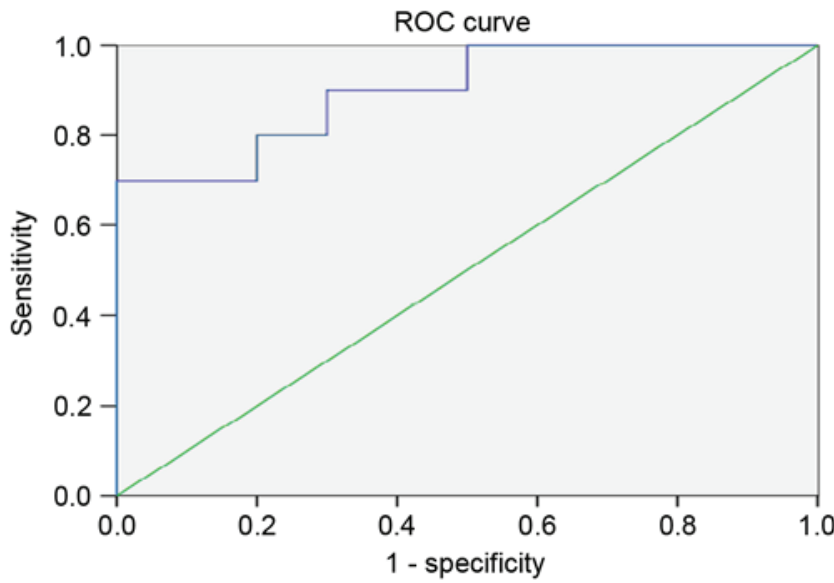

Figure 3. Area under the ROC curve for electron transfer flavoprotein $\beta$ subunit protein of 0.900 (95\% confidence interval, $0.766-1.034)$ with an optimal cutoff value of 1.59 (sensitivity $90 \%$, specificity $70 \%$ ). ROC, receiver operating characteristic.

by the gradual loss of renal energy through the development of mitochondrial dysfunction and result in increasing, oxidative stress (13).

Therefore, aerobic metabolism relies on ATP synthase along the electron transport chain (ETC) within mitochondria, which is vital for renal cellular function, yet potentially damaging in the long-term (4). The ATP5B gene encodes a $\beta$ subunit of mitochondrial ATP synthase, and the ETFB gene provides instructions for producing the $\beta$ subunit of an enzyme called electron transfer flavoprotein associated with ETC (Gene Ontology). These genes are responsible for maintaining mitochondrial membrane potential and ATP generation. ATP5B is associated with transmembrane transporter and transporter activity, and produces ATP from ADP by electron transport complexes of the respiratory chain. As for ETFB, it serves as a specific electron acceptor for several dehydrogenases, and transfers the electrons to the primary mitochondrial respiratory chain. The direct and indirect links between ATP5B, ETFB and mitochondria energy metabolism are emerging in multiple areas of investigation. The contribution of ATP5B in the regulation of types of cancer and of ETFB in the mutation of MADD have been investigated extensively $(6,7)$. To the best of our knowledge, no previous study has investigated the clinical effect of these metabolic markers in hydronephrosis.

In order to obtain further information about the association in the present study, RT-qPCR analysis and immunoblot-based molecular biology were used to investigate the differential expression in gene and protein levels between the hydronephrotic kidney tissues and the normal kidney tissues. The resulting data indicated that ATP5B and ETFB were well represented in the kidney, and they are upregulated, compared with those in the normal kidney, revealed by immunoblotting In addition, the results of the RT-qPCR analysis revealed the results of the gene levels were consistent with those of protein levels in ATP5B only. The inconsistency between the mRNA and protein expression for ETFB may be caused by posttranscriptional regulation.

In the present study, the levels ofATP5B and ETFB were significantly elevated in patients who had developed an obstructed kidney and undergone pyeloplasty. The mechanism 
underlying this increase in the patients remains to be elucidated As it has been documented that cell apoptosis is important in renal damage in hydronephrosis, and the number of apoptotic cells increases with an increasing degree of hydronephrosis (14), the present study hypothesized that the ATP5B and ETFB metabolic markers are essential in cellular energetic metabolism and the execution of cell apoptosis. Higher levels of ATP5B and ETFB are molecularly and functionally integrated to provide energy and promote the execution of apoptosis more effectively, and function in cell injury to the obstructed kidneys. Furthermore, in our previous animal model study, it was found that ATP5B was continuously upregulated and ETFB was continuously downregulated with obstruction persisting in the obstructed kidney, whereas in advanced obstruction, no significant differences were found in the expression of ETFB, compared with the sham group (Data submission). Based on data from animals and patients, the present study hypothesized that the patients in the present study, who had undergone pyeloplasty at SFU grades III and IV are in relatively early obstruction. If obstruction is relieved at this time-point, it may contribute to the long-term recovery of renal function and avoid irreversible kidney fibrosis. This hypothesis is consistent with the observations in the postoperative follow-up in the present study (data not shown). Additionally, based on data from Hirokawa et al $(15,16)$, ETFB is involved in the mechanoregulation of fibroblast cell number and the knock down of ETFB can reduce transforming growth factor- $\beta$-induced mRNA expression of $\alpha$-smooth muscle actin, and affect the tissue remodeling and/or fibrotic processes. The upregulation of ETFB may be an attempt to promote and aggravate renal fibrosis in the early stages.

The present study further evaluated the usefulness of ATP5B and ETFB in renal injury caused by obstructive nephropathy. By calculating the correlations of ATP5B and ETFB with the primary clinical features of the affected kidney, significant negative correlations were found between the protein levels of ATP5B, ETFB and the SRF, suggesting the value for ATP5B and ETFB in predicting SRF in congenital hydronephrosis. That is, the higher the expression, the lower the SRF. No significant correlations with the age of the patients, GFR, renal thickness or the anterior-posterior diameter (length) of the pelvis were found (data not shown). The present study hypothesized that ATP5B and ETFB proteins may indicate the degree of renal injury, and may represent a measurable index of renal injury, reflected by SRF, to assess whether the injury may progress to chronic kidney disease. This hypothesis was confirmed by data from the ROC analyses, which showed a useful diagnostic profile for the ETFB protein in detecting kidney injury in children with hydronephrosis with SRF $<45 \%$ (AUC, 0.900). These results suggest that the ETFB protein may be useful in identifying kidney injury.

As circulating proteins also contribute to urinary levels, whether urinary levels can also reliably measure renal injury and the impairment of renal function requires further investigation. For this, the expression levels of ETFB in the plasma or urine, and their diagnostic implications on renal injury, require assessment.

In conclusion, the results of the present pilot study preliminary demonstrated that the children in the cohort with hydronephrosis had increased gene and protein levels of ATP5B and protein levels of ETFB, based on protein in renal tissues. The protein expression levels of the markers correlated negatively with SFR in the radionuclide scan, and the ETFB protein was indicated as potentially being more useful in identifying kidney injury. The results of the present study suggested that increasing levels of metabolic markers were associated with worsening renal injury. ATP5B and ETFB may be novel diagnostic or prognostic markers, and require further detailed investigation.

\section{Acknowledgements}

This study was supported by the National Nature Science Foundation of China (grant no. 81370772).

\section{References}

1. Peters CA and Chevalier RL: Congenital urinary obstruction: Pathophysiology and clinical evaluation. In: Campbell's Urology. Kavoussi LR, Novick AC, Partin AW and Peters CA (eds). 10th edition. Saunders, Philadelphia, PA, pp3030-3047, 2012.

2. Klahr S and Morrissey J: Obstructive nephropathy and renal fibrosis. Am J Physiol Renal Physiol 283: F861-F875, 2002.

3. Mizuguchi Y, Chen J, Seshan SV, Poppas DP, Szeto HH and Felsen D: A novel cell-permeable antioxidant peptide decreases renal tubular apoptosis and damage in unilateral ureteral obstruction. Am J Physiol Renal Physiol 295: F1545-F1553, 2008.

4. Small DM, Coombes JS, Bennett N, Johnson DW and Gobe GC Oxidative stress, anti-oxidant therapies and chronic kidney disease. Nephrology (Carlton) 17: 311-321, 2012.

5. Zhang G, Oldroyd SD, Huang LH, Yang B, Li Y, Ye R and El Nahas AM: Role of apoptosis and Bcl-2/Bax in the development of tubulointerstitial fibrosis during experimental obstructive nephropathy. Exp Nephrol 9: 71-80, 2001.

6. Hjerpe E, Egyhazi Brage S, Carlson J, Frostvik Stolt M, Schedvins K, Johansson H, Shoshan M and Avall-Lundqvist E: Metabolic markers GAPDH, PKM2, ATP5B and BEC-index in advanced serous ovarian cancer. BMC Clin Pathol 13: 30, 2013.

7. Schiff M, Froissart R, Olsen RK, Acquaviva C and Vianey-Saban C: Electron transfer flavoprotein deficiency: Functional and molecular aspects. Mol Genet Metab 88: 153-158, 2006.

8. Livak KJ and Schmittgen TD: Analysis of relative gene expression data using real-time quantitative PCR and the 2(-Delta Delta C(T)) Method. Methods 25: 402-408, 2001.

9. Kajbafzadeh AM, Elmi A, Talab SS, Emami H, Esfahani SA and Saeedi P: Urinary and serum carbohydrate antigen 19-9 as a biomarker in ureteropelvic junction obstruction in children. J Urol 183: 2353-2360, 2010.

10. Chevalier RL: Obstructive nephropathy: Towards biomarker discovery and gene therapy. Nat Clin Pract Nephrol 2: 157-168, 2006.

11. Green DR and Kroemer G: The pathophysiology of mitochondrial cell death. Science 305: 626-629, 2004.

12. Power RE, Doyle BT, Higgins D, Brady HR, Fitzpatrick JM and Watson RW: Mechanical deformation induced apoptosis in human proximal renal tubular epithelial cells is caspase dependent. J Urol 171: 457-461, 2004

13. Manucha W, Carrizo L, Ruete C, Brady HR, Fitzpatrick JM and Watson RW: Angiotensin II type I antagonist on oxidative stress and heat shock protein 70 (HSP 70) expression in obstructive nephropathy. Cell Mol Biol (Noisy-le-grand) 51: 547-555, 2005.

14. Yang Y, Ji S, Wang C and Hou Y: Apoptosis of renal tubular cells in congenital hydronephrosis. Chin Med J (Engl) 114: 502-505, 2001.

15. Hirokawa S, Shimanuki T, Kitajima H, Nishimori $Y$ and Shimosaka M: Knockdown of electron transfer flavoprotein $\beta$ subunit reduced TGF- $\beta$-induced $\alpha$-SMA mRNA expression but not COL1A1 in fibroblast-populated three-dimensional collagen gel cultures. J Dermatol Sci 68: 179-186, 2012.

16. Hirokawa S, Shimanuki T, Kitajima H, Nishimori Y and Shimosaka M: Identification of ETFB as a candidate protein that participates in the mechanoregulation of fibroblast cell number in collagen gel culture. J Dermatol Sci 64: 119-126, 2011. 